\title{
Property Map by Electron Spectroscopy Imaging Series
}

\author{
Fu-Rong Chen ${ }^{1,2,3}$, Ji-Jung Kai ${ }^{1}$, Juhn-Jong Lin ${ }^{4}$, Li. Chang ${ }^{5}$ and Ray Egerton ${ }^{6}$ \\ 1Department of Engineering and System Science, National Tsing Hua University, HsinChu , \\ Taiwan. \\ 2National Synchrotron Radiation Research Center, HsinChu, Taiwan. \\ 3 Institute of Physics, Academic Sinica, Taiwan. \\ 4Department of Electrophysics, National Chiao Tung University, HsinChu, \\ Taiwan. \\ 5Department of Materials Science and Engineering, National Chiao-Tung University \\ HsinChu , Taiwan. \\ 6Department of physics, University of Alberta, Edmonton, Canada T6G 2J1.
}

Electron energy loss spectroscopy (EELS) provides powerful method to quantitative characterization of important property of materials such as composition, energy band gap, dielectric function, chemical bonding and electronic structure. In order to reveal in-homogeneity in the material, two dimensional EELS information with high spatial resolution is required. Two spectroscopic imaging methods have been developed to implement 2-D EELS. The first technique, known as the spectrum imaging method, is to acquire a 2-dimensional EELS spectrum by scanning the focused electron beam across the sample. This method was proposed by Jeanguillaume and Colliex in 1989 [1]. The other method is known as electron spectroscopic imaging (ESI) or image spectroscopy, which extracts the spectrum at a specific position from a series of energy loss images [2]. The advantage of the spectrum imaging method is that the energy resolution, which is about $0.3 \sim 1 \mathrm{eV}$ depending on the type of electron gun and the accelerating voltage, is better than for electron spectroscopic imaging (ESI) which is $4-20 \mathrm{eV}$ depending on the width of the energy-selecting slit. However, the drawbacks of spectrum imaging are the radiation damage of beam sensitive materials and a longer acquisition time than required by the ESI method [1].

Bottlenecks in the ESI method for quantitative analysis are under-sampling and loss of energy resolution, since the energy loss image series are acquired with finite size of energy slit and discrete energy step. A set of signal processing methods has been successfully developed for process electron spectroscopic images (ESI) series. In my talk, I will demonstrated the application cases of ESI to mapping of $\mathrm{sp}_{2} / \mathrm{sp}_{3}$ ratio [3], dielectric function[4], energy bandgap[5], valence state[6]. Figure 1 shows a zero loss image of $\mathrm{Cu} / \mathrm{Fe} / \mathrm{Fe}_{2} \mathrm{O}_{3}$ thin film. The ESI image series was acquired with $4 \mathrm{eV}$ energy window and $2 \mathrm{eV}$ step. Typical spectra extracted from the Fe and $\mathrm{Fe}_{2} \mathrm{O}_{3}$ thin films are shown in Fig. 2(a) and (b). The L2/L3 are clearly revealed for both cases. The resultant L2/L3 map is shown in the Fig. 3.

This work was supported by the Taiwan National Science Council through Grant Nos.

NSC 93-2120-M-009-009 and NSC 94-2120-M-009-010. 


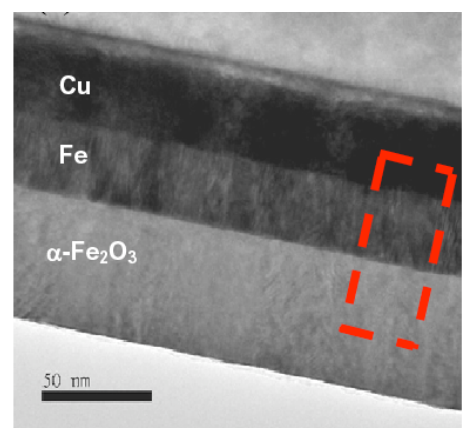

Fig. 1. A zero loss image of $\mathrm{Cu} / \mathrm{Fe} / \mathrm{Fe}_{2} \mathrm{O}_{3}$ thin film
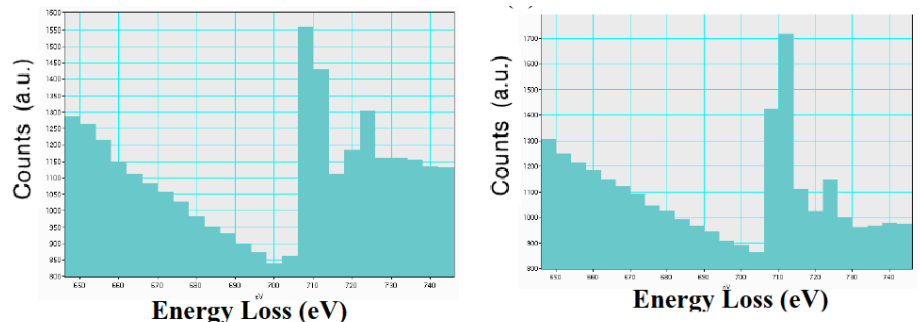

Fig. 2 the retrieved spectrum from (a) $\mathrm{Fe}$ and (b) $\mathrm{Fe}_{2} \mathrm{O}_{3}$ thin film

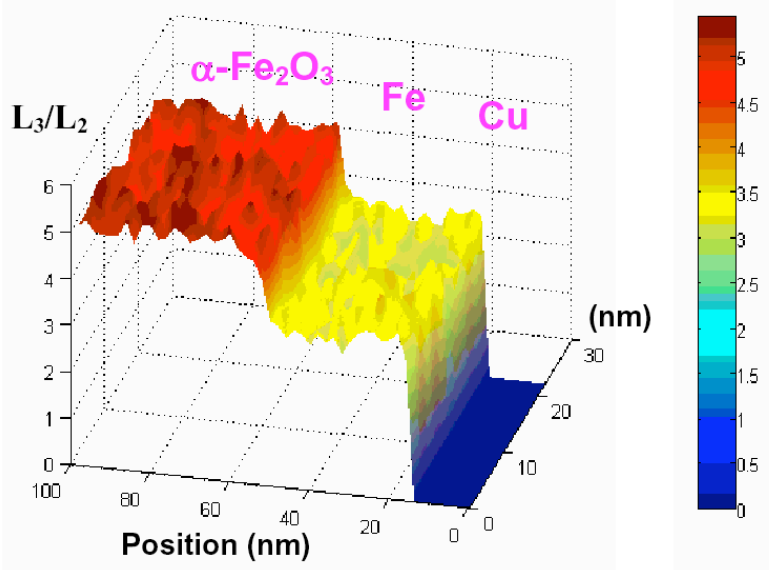

Fig. 3 the L2/L3 valence state map of the red boxed area in Fig. 1

\section{References}

1 Jeanguillaume C and Colliex C (1989) Spectrum-image: the next step in EELS digital acquisition and processing. Ultramicroscopy 78: 252-257.

2 Jeanguillaume C, Trebbia P and Colliex C (1978) About the use of electron energy-loss spectroscopy for chemical mapping of thin foils with high spatial resolution. Ultramicroscopy 3: 237242.

3. J-Y, Chen F-R and Kai J-J (2002) Mapping of sp2/sp3 in DLC thin film by signal processed ESI series energy-loss image. J. Electron Microsc. 51: 391-400.

4. Lo S-C, Kai J-J, Chen F-R, Chen L-C, Chang L, Chang C-C, Ding P-J, Chin B, Zhang H and Chen F-S (2001) Four-dimension dielectric property image obtain from electron spectroscopic imaging series. J. Electron Microsc. 50: 497-507.

5. Tsai J-S, Kai J-J, Chang L and Chen F-R (2004) Bandgap mapping for III-V quantum well by electron spectroscopy imaging. J. Electron Microsc. 53: 371-380. 